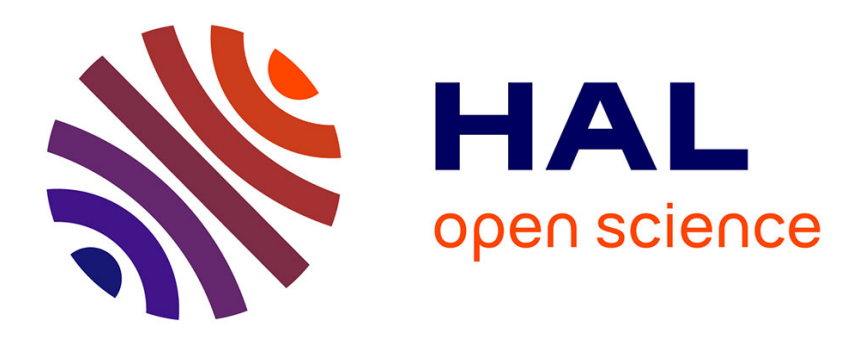

\title{
On the problem of Pillai with Padovan numbers and powers of 3
}

\author{
Mahadi Ddamulira
}

\section{To cite this version:}

Mahadi Ddamulira. On the problem of Pillai with Padovan numbers and powers of 3. Studia Scientiarum Mathematicarum Hungarica, 2019, 56 (3), pp.364-379. 10.1556/012.2019.56.3.1435 . hal02139943

\section{HAL Id: hal-02139943 \\ https://hal.science/hal-02139943}

Submitted on 26 May 2019

HAL is a multi-disciplinary open access archive for the deposit and dissemination of scientific research documents, whether they are published or not. The documents may come from teaching and research institutions in France or abroad, or from public or private research centers.
L'archive ouverte pluridisciplinaire HAL, est destinée au dépôt et à la diffusion de documents scientifiques de niveau recherche, publiés ou non, émanant des établissements d'enseignement et de recherche français ou étrangers, des laboratoires publics ou privés. 


\title{
ON THE PROBLEM OF PILLAI WITH PADOVAN NUMBERS AND POWERS OF 3
}

\author{
MAHADI DDAMULIRA
}

\begin{abstract}
Let $\left\{P_{n}\right\}_{n \geq 0}$ be the sequence of Padovan numbers defined by $P_{0}=0$ $P_{1}=1, P_{2}=1$ and $P_{n+3}=P_{n+1}+P_{n}$ for all $n \geq 0$. In this paper, we find all integers $c$ admitting at least two representations as a difference between a Padovan number and a power of 3 .
\end{abstract}

\section{INTRODUCTION}

We consider the sequence $\left\{P_{n}\right\}_{n \geq 0}$ of Padovan numbers defined by

$$
P_{0}=0, P_{1}=1, P_{2}=1 \text {, and } P_{n+3}=P_{n+1}+P_{n} \text { for all } n \geq 0 \text {. }
$$

This is sequence $A 000931$ on the Online Encyclopedia of Integer Sequences (OEIS). The first few terms of this sequence are

$$
\left\{P_{n}\right\}_{n \geq 0}=0,1,1,1,2,2,3,4,5,7,9,12,16,21,28,37,49,65,86,114,151, \ldots
$$

In this paper, we study the Diophantine equation

$$
P_{n}-3^{m}=c
$$

for a fixed integer $c$ and variable integers $n$ and $m$. In particular, we are interested in finding those integers $c$ admitting at least two representations as a difference between a Padovan number and a power of 3 . This equation is a variation of the Pillai equation

$$
a^{x}-b^{y}=c
$$

where $x, y$ are non-gative integers and $a, b, c$ are fixed positive integers.

In the 1930's, Pillai (see [19, 20]) conjectured that for any given integer $c \geq 1$, the number of positive integer solutions $(a, b, x, y)$, with $x \geq 2$ and $y \geq 2$ to the equation (2) is finite. This conjecture is still open for all $c \neq 1$. The case $c=1$ is Catalan's conjecture which was proved by Mihăilescu (see [18]). Pillai's work was an extension of the work of Herschfeld (see [15, 16 ), who had already studied a particular case of the problem with $(a, b)=(2,3)$. Since then, different variations of the Pillai equation have been studied. Some recent results for the different variations of the Pillai problem involving Fibonacci numbers, Tribonacci numbers, Pell numbers, the $k$-generalized Fibonacci numbers and other generalized linearly recurrent sequences, with powers of 2 , have been completely studied, for example, in [3, 4, 5, 6, 7, 8, 9, 10, 11, 12.

2010 Mathematics Subject Classification. 11B39, 11J86.

Key words and phrases. Padovan numbers; Linear forms in logarithms; Baker's method. 


\section{MAin RESUlT}

We discard the situations when $n=1$ and $n=2$ and just count the solutions for $n=3$ since $P_{1}=P_{2}=P_{3}=1$. The reason for the above convention is to avoid trivial parametric families such as $1-3^{m}=P_{1}-3^{m}=P_{2}-3^{m}=P_{3}-3^{m}$. For the same reasons, we discard the situation when $n=4$ and just count the solutions for $n=5$ since $P_{4}=P_{5}=2$. Thus, we always assume that $n \geq 2$ and $n \neq 4$. The main aim of this paper is to prove the following result.

Theorem 1. The only integers $c$ having at least two representations of the form $P_{n}-3^{m}$ are $c \in\{-6,0,1,22,87\}$. Furthermore, all the representations of the above integers as $P_{n}-3^{m}$ with integers $n \geq 3, n \neq 4$ and $m \geq 0$ are given by

$$
\begin{aligned}
-6 & =P_{13}-3^{3}=P_{6}-3^{2} ; \\
0 & =P_{10}-3^{2}=P_{6}-3^{1}\left(=P_{3}-3^{0}\right) ; \\
1 & =P_{14}-3^{3}=P_{7}-3^{1}\left(=P_{5}-3^{0}\right) ; \\
22 & =P_{20}-3^{5}=P_{16}-3^{3} ; \\
87 & =P_{24}-3^{6}=P_{17}-3^{3} .
\end{aligned}
$$

\section{Preliminary Results}

3.1. The Padovan sequence. Here, we recall some important properties of the Padovan sequence $\left\{P_{n}\right\}_{n \geq 0}$. The characteristic equation

$$
\Psi(x):=x^{3}-x-1=0
$$

has $\operatorname{roots} \alpha, \beta, \gamma=\bar{\beta}$, where

$$
\alpha=\frac{r_{1}+r_{2}}{6}, \quad \beta=\frac{-\left(r_{1}+r_{2}\right)+\sqrt{-3}\left(r_{1}-r_{2}\right)}{12}
$$

and

$$
r_{1}=\sqrt[3]{108+12 \sqrt{69}} \text { and } r_{2}=\sqrt[3]{108-12 \sqrt{69}}
$$

Furthermore, the Binet formula is given by

$$
P_{n}=a \alpha^{n}+b \beta^{n}+c \gamma^{n} \quad \text { for all } \quad n \geq 0,
$$

where

$$
a=\frac{(1-\beta)(1-\gamma)}{(\alpha-\beta)(\alpha-\gamma)}, \quad b=\frac{(1-\alpha)(1-\gamma)}{(\beta-\alpha)(\beta-\gamma)}, \quad c=\frac{(1-\alpha)(1-\beta)}{(\gamma-\alpha)(\gamma-\beta)}=\bar{b} .
$$

Numerically, the following estimates hold:

$$
\begin{gathered}
1.32<\alpha<1.33 \\
0.86<|\beta|=|\gamma|=\alpha^{-\frac{1}{2}}<0.87 \\
0.72<a<0.73 \\
0.24<|b|=|c|<0.25 .
\end{gathered}
$$

By induction, one can easily prove that

$$
\alpha^{n-2} \leq P_{n} \leq \alpha^{n-1} \text { holds for all } n \geq 4 .
$$


Let $\mathbb{K}:=\mathbb{Q}(\alpha, \beta)$ be the splitting field of the polynomial $\Psi$ over $\mathbb{Q}$. Then $[\mathbb{K}, \mathbb{Q}]=6$. Furthermore, $[\mathbb{Q}(\alpha): \mathbb{Q}]=3$. The Galois group of $\mathbb{K}$ over $\mathbb{Q}$ is given by

$$
\mathcal{G}:=\operatorname{Gal}(\mathbb{K} / \mathbb{Q}) \cong\{(1),(\alpha \beta),(\alpha \gamma),(\beta \gamma),(\alpha \beta \gamma),(\alpha \gamma \beta)\} \cong S_{3}
$$

Thus, we identify the automorphisms of $\mathcal{G}$ with the permutations of the roots of the polynomial $\Psi$. For example, the permutation $(\alpha \gamma)$ corresponds to the automorphism $\sigma: \alpha \rightarrow \gamma, \gamma \rightarrow \alpha, \beta \rightarrow \beta$.

3.2. Linear forms in logarithms. To prove our main result Theorem 1 we use several times a Baker-type lower bound for a nonzero linear form in logarithms of algebraic numbers. There are many such bounds in the literature like that of Baker and Wüstholz from [2]. In this paper we use the result of Matveev [17, which is one of our main tools. Let $\gamma$ be an algebraic number of degree $d$ with minimal primitive polynomial over the integers

$$
a_{0} x^{d}+a_{1} x^{d-1}+\cdots+a_{d}=a_{0} \prod_{i=1}^{d}\left(x-\gamma^{(i)}\right),
$$

where the leading coefficient $a_{0}$ is positive and the $\eta^{(i)}$ 's are the conjugates of $\gamma$. Then the logarithmic height of $\gamma$ is given by

$$
h(\gamma):=\frac{1}{d}\left(\log a_{0}+\sum_{i=1}^{d} \log \left(\max \left\{\left|\gamma^{(i)}\right|, 1\right\}\right)\right) .
$$

In particular, if $\gamma=p / q$ is a rational number with $\operatorname{gcd}(p, q)=1$ and $q>0$, then $h(\gamma)=\log \max \{|p|, q\}$. The following are some of the properties of the logarithmic height function $h(\cdot)$, which will be used in the next sections of this paper without reference:

$$
\begin{aligned}
h(\eta \pm \gamma) & \leq h(\eta)+h(\gamma)+\log 2, \\
h\left(\eta \gamma^{ \pm 1}\right) & \leq h(\eta)+h(\gamma), \\
h\left(\eta^{s}\right) & =|s| h(\eta)(s \in \mathbb{Z}) .
\end{aligned}
$$

Theorem 2 (Matveev). Let $\gamma_{1}, \ldots, \gamma_{t}$ be positive real algebraic numbers in a real algebraic number field $\mathbb{K}$ of degree $D, b_{1}, \ldots, b_{t}$ be nonzero integers, and assume that

$$
\Lambda:=\gamma_{1}^{b_{1}} \cdots \gamma_{t}^{b_{t}}-1
$$

is nonzero. Then

$$
\log |\Lambda|>-1.4 \times 30^{t+3} \times t^{4.5} \times D^{2}(1+\log D)(1+\log B) A_{1} \cdots A_{t},
$$

where

and

$$
B \geq \max \left\{\left|b_{1}\right|, \ldots,\left|b_{t}\right|\right\}
$$

$$
A_{i} \geq \max \left\{D h\left(\gamma_{i}\right),\left|\log \gamma_{i}\right|, 0.16\right\}, \quad \text { for all } \quad i=1, \ldots, t .
$$

3.3. Baker-Davenport reduction lemma. During the calculations, we get upper bounds on our variables which are too large, thus we need to reduce them. To do so, we use some results from the theory of continued fractions. Specifically, for a nonhomogeneous linear form in two integer variables, we use a slight variation of a result due to Dujella and Pethö (see [13, Lemma 5a), which is itself a generalization of a result of Baker and Davenport [1]. For a real number $X$, we write $\|X\|:=\min \{|X-n|: n \in \mathbb{Z}\}$ for the distance from $X$ to the nearest integer. 
Lemma 1 (Dujella, Pethö). Let $M$ be a positive integer, $p / q$ be a convergent of the continued fraction of the irrational number $\tau$ such that $q>6 M$, and $A, B, \mu$ be some real numbers with $A>0$ and $B>1$. Let further $\varepsilon:=\|\mu q\|-M\|\tau q\|$. If $\varepsilon>0$, then there is no solution to the inequality

$$
0<|u \tau-v+\mu|<A B^{-w},
$$

in positive integers $u, v$ and $w$ with

$$
u \leq M \quad \text { and } \quad w \geq \frac{\log (A q / \varepsilon)}{\log B} .
$$

Finally, the following lemma is also useful. It is Lemma 7 in [14.

Lemma 2 (Gúzman, Luca). If $m \geqslant 1, Y>\left(4 m^{2}\right)^{m}$ and $Y>x /(\log x)^{m}$, then

$$
x<2^{m} Y(\log Y)^{m} \text {. }
$$

\section{Proof of Theorem 1}

Assume that there exist positive integers $n, m, n_{1}, m_{1}$ such that $(n, m) \neq\left(n_{1}, m_{1}\right)$, and

$$
P_{n}-3^{m}=P_{n_{1}}-3^{m_{1}} .
$$

In particular, we can assume that $m \geq m_{1}$. If $m=m_{1}$, then $P_{n}=P_{n_{1}}$, so $(n, m)=$ $\left(n_{1}, m_{1}\right)$, which gives a contradiction to our assumption. Thus $m>m_{1} \geq 0$. Since

$$
P_{n}-P_{n_{1}}=3^{m}-3^{m_{1}},
$$

and the right-hand side is positive, we get that the left-hand side is also positive and so $n>n_{1}$. Thus, $n \geq 5$ and $n_{1} \geq 3$, because $n \neq 4$.

Using the equation (10) and the inequality 7 , we get

$$
\begin{aligned}
& \alpha^{n-4} \leq P_{n-2} \leq P_{n}-P_{n_{1}}=3^{m}-3^{m_{1}}<3^{m}, \\
& \alpha^{n-1} \geq P_{n} \geq P_{n}-P_{n_{1}}=3^{m}-3^{m_{1}} \geq 3^{m-1},
\end{aligned}
$$

from which we get that

$$
1+\left(\frac{\log 3}{\log \alpha}\right)(m-1)<n<\left(\frac{\log 3}{\log \alpha}\right) m+4 .
$$

If $n<500$, then $m \leq 200$. We ran a Mathematica program for $2 \leq n_{1}<n \leq 500$ and $0 \leq m_{1}<m \leq 200$ and found only the solutions from the list (3). From now, we assume that $n \geq 500$. Note that the inequality $(13)$ implies that $4 m<n$. Therefore, to solve the Diophatine equation (1), it suffices to find an upper bound for $n$.

4.1. Bounding $n$. By using (1) and (4) and the estimates (6), we get

$$
\begin{aligned}
a \alpha^{n}+b \beta^{n}+c \gamma^{n}-3^{m} & =a \alpha^{n_{1}}+b \beta^{n_{1}}+c \gamma^{n_{1}}-3^{m_{1}} \\
\left|a \alpha^{n}-3^{m}\right| & =\left|a \alpha^{n_{1}}+b\left(\beta^{n_{1}}-\beta^{n}\right)+c\left(\gamma^{n_{1}}-\gamma^{n}\right)-3^{m_{1}}\right| \\
& \leq a \alpha^{n_{1}}+|b|\left(|\beta|^{n}+|\beta|^{n_{1}}\right)+|c|\left(|\gamma|^{n}+|\gamma|^{n_{1}}\right)+3^{m_{1}} \\
& \leq a \alpha^{n_{1}}+2|b|\left(|\beta|^{n}+|\beta|^{n_{1}}\right)+3^{m_{1}} \\
& \leq a \alpha^{n_{1}}+4|b||\beta|^{n}+3^{m_{1}} \\
& <\alpha^{n_{1}}+3^{m_{1}} \\
& <2 \max \left\{\alpha^{n_{1}}, 3^{m_{1}}\right\}
\end{aligned}
$$


Multiplying through by $3^{-m}$, using the relation (11) and using the fact that $\alpha<3$, we get

$$
\left|a \alpha^{n} 3^{-m}-1\right|<2 \max \left\{\frac{\alpha^{n_{1}}}{3^{m}}, 3^{m_{1}-m}\right\}<\max \left\{\alpha^{n_{1}-n+5}, 3^{m_{1}-m+1}\right\} .
$$

For the left-hand side, we apply the result of Matveev, Theorem 2 with the following data

$$
t=3, \gamma_{1}=a, \gamma_{2}=\alpha, \gamma_{3}=3, b_{1}=1, b_{2}=n, b_{3}=-m \text {. }
$$

Through out we work with the field $\mathbb{K}:=\mathbb{Q}(\alpha)$ with $D=3$. Since $\max \{1, n, m\} \leq n$, we take $B:=n$. Further,

$$
a=\frac{\alpha(\alpha+1)}{3 \alpha^{2}-1}
$$

the minimum polynomial of $a$ is $23 x^{3}-23 x^{2}+6 x-1$ and has roots $a, b, c$. Also by (6), we have $\max \{|a|,|b|,|c|\}<1$. Thus, $h\left(\gamma_{1}\right)=h(a)=\frac{1}{3} \log 23$. So we can take $A_{1}:=3 h\left(\gamma_{1}\right)=\log 23$. We can also take $A_{2}:=3 h\left(\gamma_{2}\right)=\log \alpha, A_{3}:=3 h\left(\gamma_{3}\right)=3 \log 3$. We put

$$
\Lambda=a \alpha^{n} 3^{-m}-1 .
$$

First we check that $\Lambda \neq 0$, if it were, then $a \alpha^{n}=3^{m} \in \mathbb{Z}$. Conjugating this relation by the automorphism $(\alpha \beta)$, we obtain that $b \beta^{n}=3^{m}$, which is a contradiction because $\left|b \beta^{n}\right|<1$ while $3^{m} \geq 1$ for all $m \geq 0$. Thus, $\Lambda \neq 0$. Then by Matveev's theorem, the left-hand side of (14) is bounded as

$$
\log |\Lambda|>-1.4 \cdot 30^{6} \cdot 3^{4.5} \cdot 3^{2}(1+\log 3)(1+\log n)(\log 23)(\log \alpha)(3 \log 3) .
$$

By comparing with (14), we get

$$
\min \left\{\left(n-n_{1}-5\right) \log \alpha,\left(m-m_{1}-1\right) \log 3\right\}<7.97 \times 10^{12}(1+\log n),
$$

which gives

$$
\min \left\{\left(n-n_{1}\right) \log \alpha,\left(m-m_{1}\right) \log 3\right\}<7.98 \times 10^{12}(1+\log n) .
$$

Now we split the argument into two cases

Case 1. $\min \left\{\left(n-n_{1}\right) \log \alpha,\left(m-m_{1}\right) \log 3\right\}=\left(n-n_{1}\right) \log \alpha$.

In this case, we rewrite 10 as

$$
\begin{aligned}
\left|a \alpha^{n}-a \alpha^{n_{1}}-3^{m}\right| & \leq|b|\left(|\beta|^{n}+|\beta|^{n_{1}}\right)+|c|\left(|\gamma|^{n}+|\gamma|^{n_{1}}\right)+3^{m_{1}} \\
& \leq 2|b|\left(|\beta|^{n}+|\beta|^{n_{1}}\right)+3^{m_{1}} \\
& \leq 4|b||\beta|^{n}+3^{m_{1}} \\
& <1+3^{m_{1}} \leq 3^{m_{1}+1}
\end{aligned}
$$

which implies

$$
\left|a\left(\alpha^{n-n_{1}}-1\right) \alpha^{n_{1}} 3^{-m}-1\right|<3^{m_{1}-m+1} .
$$

We put

$$
\Lambda_{1}=a\left(\alpha^{n-n_{1}}-1\right) \alpha^{n_{1}} 3^{-m}-1 .
$$

As before, we take $\mathbb{K}=\mathbb{Q}(\alpha)$, so we have $D=3$. To see that $\Lambda_{1} \neq 0$, for if $\Lambda_{1}=0$, then

$$
a\left(\alpha^{n-n_{1}}-1\right) \alpha^{n_{1}}=3^{m} .
$$

By conjugating the above relation by the Galois automorphism $(\alpha \beta)$, we get that

$$
b\left(\beta^{n-n_{1}}-1\right) \beta^{n_{1}}=3^{m} .
$$


The absolute value of the left-hand side is at most $\left|b\left(\beta^{n-n_{1}}-1\right) \beta^{n_{1}-1}\right| \leq|b|\left(\left|\beta^{n}\right|+\left|\beta^{n_{1}}\right|\right)<$ $2|b||\beta|^{n}<1$, while the absolute value of the right-hand side is at least $3^{m} \geq 1$ for all $m \geq 0$, which is a contradiction. Thus, $\Lambda_{1} \neq 0$.

We apply Theorem 2 on the left-hand side of 16 with the data

$$
t=3, \gamma_{1}=a\left(\alpha^{n-n_{1}}-1\right), \gamma_{2}=\alpha, \gamma_{3}=3, b_{1}=1, b_{2}=n_{1}, b_{3}=-m .
$$

Since

$$
\begin{aligned}
h\left(\gamma_{1}\right) & \leq h(a)+h\left(\alpha^{n-n_{1}}-1\right) \\
& <\frac{1}{3} \log 23+\frac{1}{3}\left(n-n_{1}\right) \log \alpha+\log 2 \\
& <\frac{1}{3}(\log 8+\log 23)+\frac{1}{3} \times 7.98 \times 10^{12}(1+\log n) \quad \text { by } 15 \\
& <\frac{1}{3} \times 8.00 \times 10^{12}(1+\log n)
\end{aligned}
$$

So, we can take $A_{1}:=8.00 \times 10^{12}(1+\log n)$. Furthermore, as before, we take $A_{2}:=\log \alpha$ and $A_{3}:=3 \log 3$. Finally, since $\max \left\{1, n_{1}, m\right\} \leq n$, we can take $B:=n$. Then, we get

$\log \left|\Lambda_{1}\right|>-1.4 \cdot 30^{6} \cdot 3^{4.5} \cdot 3^{2}(1+\log 3)(1+\log n)\left(8.00 \times 10^{12}(1+\log n)\right)(\log \alpha)(3 \log 3)$.

Then,

$$
\log \left|\Lambda_{1}\right|>-6.38 \times 10^{25}(1+\log n)^{2} .
$$

By comparing the above relation with (16), we get that

$$
\left(m-m_{1}\right) \log 3<6.40 \times 10^{25}(1+\log n)^{2} .
$$

Case 2. $\min \left\{\left(n-n_{1}\right) \log \alpha,\left(m-m_{1}\right) \log 3\right\}=\left(m-m_{1}\right) \log 3$.

In this case, we rewrite 10 as

$$
\begin{aligned}
\left|a \alpha^{n}-\left(3^{m-m_{1}}-1\right) \cdot 3^{m_{1}}\right| & \leq a \alpha^{n_{1}}+|b|\left(|\beta|^{n}+|\beta|^{n_{1}}\right)+|c|\left(|\gamma|^{n}+|\gamma|^{n_{1}}\right) \\
& \leq a \alpha^{n_{1}}+4|b||\beta|^{n} \\
& <1+\frac{3}{4} \alpha^{n_{1}}<\alpha^{n_{1}}
\end{aligned}
$$

which implies that

$$
\begin{aligned}
\left|a\left(3^{m-m_{1}}-1\right)^{-1} \alpha^{n} 3^{-m_{1}}-1\right| & <\frac{\alpha^{n_{1}}}{3^{m}-3^{m_{1}}} \leq \frac{3 \alpha^{n_{1}}}{3^{m}} \\
& <3 \alpha^{n_{1}-n+4}<\alpha^{n_{1}-n+5}
\end{aligned}
$$

We put

$$
\Lambda_{2}=a\left(3^{m-m_{1}}-1\right)^{-1} \alpha^{n} 3^{-m_{1}}-1 .
$$

Clearly, $\Lambda_{2} \neq 0$, for if $\Lambda_{2}=0$, then $a \alpha^{n}=3^{m}-3^{m_{1}}$, by similar arguments of conjugation and taking absolute values on both sides as before we get a contradiction. We again apply Theorem 2 with the following data

$$
t=3, \gamma_{1}=a\left(3^{m-m_{1}}-1\right)^{-1}, \gamma_{2}=\alpha, \gamma_{3}=\alpha, b_{1}=1, b_{2}=n, b_{3}=-m_{1} .
$$


We note that

$$
\begin{aligned}
h\left(\gamma_{1}\right) & =h\left(a\left(3^{m-m_{1}}-1\right)^{-1}\right) \leq h(a)+h\left(3^{m-m_{1}}-1\right) \\
& =\frac{1}{3} \log 23+h\left(3^{m-m_{1}}-1\right)<\log \left(3^{m-m_{1}+2}\right) \\
& \left.=\left(m-m_{1}+2\right) \log 3<8.00 \times 10^{13}(1+\log n) \text { by } 15\right) .
\end{aligned}
$$

So, we can take $A_{1}:=2.40 \times 10^{13}(1+\log n)$. Further, as in the previous applications, we take $A_{2}:=\log \alpha$ and $A_{3}:=3 \log 3$. Finally, since $\max \left\{1, n, m_{1}\right\} \leq n$, we can take $B:=n$. Then, we get

$\log \left|\Lambda_{2}\right|>-1.4 \cdot 30^{6} \cdot 3^{4.5} \cdot 3^{2}(1+\log 3)(1+\log n)\left(2.40 \times 10^{13}(1+\log n)\right)(\log \alpha)(3 \log 3)$.

Thus,

$$
\log \left|A_{2}\right|>-1.91 \times 10^{26}(1+\log n)^{2} .
$$

Now, by comparing with 19 , we get that

$$
\left(n-n_{1}\right) \log \alpha<1.92 \times 10^{26}(1+\log n)^{2} .
$$

Therefore, in both Case 1 and Case 2, we have

$$
\begin{aligned}
\min \left\{\left(n-n_{1}\right) \log \alpha,\left(m-m_{1}\right) \log 3\right\} & <7.98 \times 10^{12}(1+\log n), \\
\max \left\{\left(n-n_{1}\right) \log \alpha,\left(m-m_{1}\right) \log 3\right\} & <1.92 \times 10^{26}(1+\log n)^{2} .
\end{aligned}
$$

Finally, we rewrite the equation 10 as

$$
\left|a \alpha^{n}-a \alpha^{n_{1}}-3^{m}+3^{m_{1}}\right|=\left|b \beta^{n_{1}}+c \gamma^{n_{1}}\right|<1 .
$$

Dividing through by $3^{m}-3^{m_{1}}$, we get

$$
\begin{aligned}
\left|\frac{a\left(\alpha^{n-n_{1}}-1\right)}{3^{m-m_{1}}-1} \alpha^{n_{1}} 3^{-m_{1}}-1\right| & <\frac{1}{3^{m}-3^{m_{1}}} \leq \frac{3}{3^{m}} \\
& \leq 3 \alpha^{-\left(n+n_{1}-4\right)} \leq \alpha^{4-n}
\end{aligned}
$$

since $1.32<\alpha \leq \alpha^{n_{1}}$. We again apply Theorem 2 on the left-hand side of 22 with the data

$$
t=3, \gamma_{1}=\frac{a\left(\alpha^{n-n_{1}}-1\right)}{3^{m-m_{1}}-1}, \gamma_{2}=\alpha, \gamma_{3}=3, b_{1}=1, b_{2}=n_{1}, b_{3}=-m_{1}
$$

By using the algebraic properties of the logarithmic height function, we get

$$
\begin{aligned}
3 h\left(\gamma_{1}\right) & =3 h\left(\frac{a\left(\alpha^{n-n_{1}}-1\right)}{3^{m-m_{1}}-1}\right) \leq 3 h\left(a\left(\alpha^{n-n_{1}}-1\right)\right) 3+h\left(3^{m-m_{1}}-1\right) \\
& <\log 23+3 \log 2+3\left(n-n_{1}\right) \log \alpha+3\left(m-m_{1}\right) \log 3 \\
& <3.86 \times 10^{26}(1+\log n)^{2},
\end{aligned}
$$

where in the above inequalities, we used the argument from (21). Thus, we can take $A_{1}:=3.86 \times 10^{26}(1+\log n)$, and again as before $A_{2}:=\log \alpha$ and $A_{3}:=3 \log 3$. If we put

$$
\Lambda_{3}=\frac{a\left(\alpha^{n-n_{1}}-1\right)}{3^{m-m_{1}}-1} \alpha^{n_{1}} 3^{-m_{1}}-1,
$$

we need to show that $\Lambda_{3} \neq 0$. If not, $\Lambda_{3}=0$ leads to

$$
a\left(\alpha^{n}-\alpha^{n_{1}}\right)=3^{m}-3^{m_{1}} .
$$


A contradiction is reached upon a conjuagtion by the automorphism $(\alpha \beta)$ in $\mathbb{K}$ and by taking absolute values on both sides. Thus, $\Lambda_{3} \neq 0$. Applying Theorem 2 gives

$\log \left|\Lambda_{3}\right|>-1.4 \cdot 30^{6} \cdot 3^{4.5} \cdot 3^{2}(1+\log 3)(1+\log n)\left(3.86 \times 10^{26}(1+\log n)^{2}\right)(\log \alpha)(3 \log 3)$, a comparison with 22 gives

$$
(n-4)<3.08 \times 10^{39}(1+\log n)^{3},
$$

or

$$
n<3.10 \times 10^{39}(1+\log n)^{3} .
$$

Now by applying Lemma 2 on 23 with the data $m=3, Y=3.10 \times 10^{39}$ and $x=n$, leads to $n<2 \times 10^{46}$.

4.2. Reducing the bound for $n$. We need to reduce the above bound for $n$ and to do so we make use of Lemma 1 several times. To begin, we return to (14) and put

$$
\Gamma:=n \log \alpha-m \log 3+\log a .
$$

For technical reasons we assume that $\min \left\{n-n_{1}, m-m_{1}\right\} \geq 20$. We go back to the inequalities for $\Lambda, \Lambda_{1}$ and $\Lambda_{2}$, Since we assume that $\min \left\{n-n_{1}, m-m_{1}\right\} \geq 20$ we get $\left|e^{\Gamma}-1\right|=|\Lambda|<\frac{1}{4}$. Hence, $|\Lambda|<\frac{1}{2}$ and since the inequality $|y|<2\left|e^{y}-1\right|$ holds for all $y \in\left(-\frac{1}{2}, \frac{1}{2}\right)$, we get

$$
0<|\Gamma|<2 \max \left\{\alpha^{n_{1}-n+5}, 3^{m_{1}-m+1}\right\} \leq \max \left\{\alpha^{n_{1}-n+6}, 3^{m_{1}-m+2}\right\} .
$$

Assume that $\Gamma>0$. We then have the inequality

$$
\begin{aligned}
n\left(\frac{\log \alpha}{\log 3}\right)-m+\frac{\log a}{\log 3} & <\max \left\{\frac{\alpha^{6}}{(\log 3) \alpha^{n-n_{1}}}, \frac{9}{(\log 3) 3^{m-m_{1}}}\right\} . \\
& <\max \left\{36 \cdot \alpha^{-\left(n-n_{1}\right)}, 9 \cdot 3^{-\left(m-m_{1}\right)}\right\} .
\end{aligned}
$$

We apply Lemma 1 with the data

$$
\tau=\frac{\log \alpha}{\log 3}, \mu=\frac{\log a}{\log 3},(A, B)=(36, \alpha) \text { or }(9,3) \text {. }
$$

Let $\tau=\left[a_{0} ; a_{1}, a_{2}, \ldots\right]=[0 ; 3,1,9,1,2,1,4,1,2,2,1,1,3,1,2,1,20,1,1,1,3,11,1, \ldots]$ be the continued fraction of $\tau$. We choose $M:=2 \times 10^{46}$ which is the upper bound on $n$. By Mathematica, we find out that the convergent

$$
\frac{p}{q}=\frac{p_{88}}{q_{88}}=\frac{3123049185137266854491675319812527194766363593581}{12201370578769620000479260876419428374896683408344}
$$

is such that $q=q_{88}>6 M$. Furthermore, it yields $\varepsilon>0.394$, and therefore either

$$
n-n_{1} \leq \frac{\log (36 q / \varepsilon)}{\log \alpha}<416, \text { or } m-m_{1} \leq \frac{\log (9 q / \varepsilon)}{\log 3}<105 .
$$

In the case $\Gamma<0$, we consider the inequality

$$
\begin{aligned}
m\left(\frac{\log 3}{\log \alpha}\right)-n+\frac{\log (1 / a)}{\log \alpha} & <\max \left\{\frac{\alpha^{6}}{\log \alpha} \alpha^{-\left(n-n_{1}\right)}, \frac{9}{\log \alpha} \cdot 3^{-\left(m-m_{1}\right)}\right\} \\
& <\max \left\{64 \alpha^{-\left(n-n_{1}\right)}, 15 \cdot 3^{-\left(m-m_{1}\right)}\right\} .
\end{aligned}
$$

We then apply Lemma 1 with the data

$$
\tau=\frac{\log 3}{\log \alpha}, \mu=\frac{\log (1 / a)}{\log \alpha},(A, B)=(64, \alpha), \text { or }(15,3) .
$$


Let $\tau=\left[a_{0} ; a_{1}, a_{2}, \ldots\right]=[3 ; 4,4,1,1,4,4,9,11,2,7,4,2,4,2,1,1,1,1,2,1,1,16,1, \ldots]$ be the continued fraction of $\tau$. Again, we choose $M=3 \times 10^{46}$, and in this case the convergent $p / q=p_{91} / q_{91}$ is such that $q=q_{91}>6 M$. Further, this yields $\varepsilon>0.394$, and therefore either

$$
n-n_{1} \leq \frac{\log (64 q / \varepsilon)}{\log \alpha}<416, \text { or } m-m_{1} \leq \frac{\log (15 q / \varepsilon)}{\log 3}<105 .
$$

These bounds agree with the bounds obtained in the case $\Gamma>0$. As a conclusion, we have that either $n-n_{1} \leq 416$ or $m-m_{1} \leq 105$ whenever $\Gamma \neq 0$.

Now, we distinguish between the cases $n-n_{1} \leq 416$ and $m-m_{1} \leq 105$. First, we assume that $n-n_{1} \leq 416$. In this case we consider the inequality for $\Lambda_{1},(16)$ and also assume that $m-m_{1} \leq 20$. We put

$$
\Gamma_{1}=n_{1} \log \alpha-m \log 3+\log \left(a\left(\alpha^{n-n_{1}}-1\right)\right) .
$$

Then inequality (16) implies that

$$
\left|\Gamma_{1}\right|<\frac{6}{3^{m-m_{1}}} .
$$

If we further assume that $\Gamma_{1}>0$, we then get

$$
0<n_{1}\left(\frac{\log \alpha}{\log 3}\right)-m+\frac{\log \left(a\left(\alpha^{n-n_{1}}-1\right)\right)}{\log 3}<\frac{6}{(\log 3) 3^{m-m_{1}}}<\frac{6}{3^{m-m_{1}}} .
$$

Again we apply Lemma 1 with the same $\tau$ as in the case $\Gamma>0$. We use the 88 -th convergent $p / q=p_{88} / q_{88}$ of $\tau$ as before. But in this case we choose $(A, B):=(9,3)$ and use

$$
\mu_{l}=\frac{\log \left(a\left(\alpha^{l}-1\right)\right)}{\log 3}
$$

instead of $\mu$ for each possible value of $l:=n-n_{1} \in[1,2, \ldots, 416]$. For all values of $l$, we get $\varepsilon>9.9954 \times 10^{-8}$. Hence by Lemma 1 , we get

$$
m-m_{1}<\frac{\log (9 q / \varepsilon)}{\log 3}<110 .
$$

Thus, $n-n_{1} \leq 416$ implies that $m-m_{1} \leq 110$. A similar conclusion is reached when $\Gamma_{1}<0$.

Now let us turn to the case $m-m_{1} \leq 105$ and we consider the inequlity for $\Lambda_{2}, 19$. We put

$$
\Gamma_{2}=n \log \alpha-m_{1} \log 3+\log \left(a\left(3^{m-m_{1}}-1\right)\right),
$$

and we also assume that $n-n_{1} \geq 20$. We then have

$$
\left|\Gamma_{2}\right|<\frac{2 \alpha^{6}}{\alpha^{n-n_{1}}} .
$$

We assume that $\Gamma_{2}$, then we get

$$
0<n\left(\frac{\log \alpha}{\log 3}\right)-m_{1}+\frac{\log \left(a\left(3^{m-m_{1}}-1\right)\right)}{\log 3}<\frac{3 \alpha^{6}}{(\log 3) \alpha^{n-n_{1}}}<\frac{106}{\alpha^{n-n_{1}}} .
$$

We apply again Lemma 1 with the same $\tau, q, M,(A, B):=(106, \alpha)$ and

$$
\mu_{l}=\frac{\log \left(a\left(3^{l}-1\right)\right)}{\log 3} \text { for } k=1,2, \ldots, 105 .
$$


We get $\varepsilon>7.7434 \times 10^{-11}$, therefore

$$
n-n_{1}<\frac{\log (106 q / \varepsilon)}{\log \alpha}<464 .
$$

A similar conclusion is reached when $\Gamma_{2}<0$. To conclude, we first get that either $n-n_{1} \leq 416$ or $m-m_{1} \leq 105$. If $n-n_{1} \leq 416$, then $m-m_{1} \leq 110$, and if $m-m_{1} \leq 105$ then $n-n_{1} \leq 464$. Thus, we conclude that we always have $n-n_{1} \leq 464$ and $m-m_{1} \leq 110$. Finally we go to the inequality of $\Lambda_{3}, 22$. We put

$$
\Gamma_{3}=n_{1} \log \alpha-m_{1} \log 3+\log \left(\frac{a\left(\alpha^{n-n_{1}}-1\right)}{3^{m-m_{1}}-1}\right) .
$$

Since $n \geq 500$, the inequality 22 implies that

$$
\left|\Gamma_{3}\right|<\frac{3}{\alpha^{n-4}}=\frac{3 \alpha^{6}}{\alpha^{n}} .
$$

Assuming that $\Gamma_{3}>0$, then

$$
0<n_{1}\left(\frac{\log \alpha}{\log 3}\right)-m_{1}+\frac{\log \left(a\left(\alpha^{k}-1\right) /\left(3^{l}-1\right)\right.}{\log 3}<\frac{3 \alpha^{6}}{(\log 3) \alpha^{n}}<\frac{116}{\alpha^{n}},
$$

where $(k, l):=\left(n-n_{1}, m-m_{1}\right)$. We again apply Lemma1 1 with the same $\tau, q, M,(A, B):=$ $(116, \alpha)$ and

$$
\mu_{k, l}=\frac{\log \left(a\left(\alpha^{k}-1\right) /\left(3^{l}-1\right)\right.}{\log 3} \text { for } 1 \leq k \leq 464,1 \leq l \leq 110 .
$$

For these cases, we get $\varepsilon>4.579572 \times 10^{-10}$, so we obtain

$$
n \leq \frac{\log (116 q / \varepsilon)}{\log \alpha}<458
$$

A similar conclusion is reached when $\Gamma_{3}<0$. Hence, $n<500$. However, this contradicts our working assumption that $n \geq 500$. This completes the proof of Theorem 1 .

\section{ACKNOWLEDGEMENTS}

The author was supported by the Austrian Science Fund (FWF) grants: F5510-N26 Part of the special research program (SFB), "Quasi Monte Carlo Metods: Theory and Applications" and W1230 - "Doctoral Program Discrete Mathematics". This paper was written when the author visited the Institut de Mathématiques de Bordeaux, Université de Bordeaux, in May 2019. He would like to thank this institution for its hospitality and the fruitful working environment.

\section{REFERENCES}

[1] A. Baker and H. Davenport. The equations $3 x^{2}-2=y^{2}$ and $8 x^{2}-7=z^{2}$. The Quarterly Journal of Mathematics, 20(1):129-137, 1969.

[2] A. Baker and G. Wüstholz. Logarithmic forms and Diophantine geometry, volume 9. Cambridge University Press, 2008.

[3] J. J. Bravo, F. Luca, and K. Yazán. On Pillai's problem with Tribonacci numbers and powers of 2. Bulletin of Korean Mathematical Society 54(3):1069-1080, 2017.

[4] K. C. Chim, I. Pink and V. Ziegler. On a variant of Pillai's problem. International Journal of Number Theory, 13(7):1711-1727, 2017.

[5] K. C. Chim, I. Pink and V. Ziegler. On a variant of Pillai's problem II. Journal of Number Theory, 183:269-290, 2018.

[6] M. Ddamulira. On the problem of Pillai with Fibonacci numbers and powers of 3. Preprint, 2019. 
[7] M. Ddamulira. On the problem of Pillai with Tribonacci numbers and powers of 3. Preprint, 2019.

[8] M. Ddamulira and F. Luca. On a problem of Pillai with $k$-generalized Fibonacci numbers and powers of 3. Preprint, 2019

[9] M. Ddamulira, F. Luca and M. Rakotomalala. On a problem of Pillai with Fibonacci numbers and powers of 2. Proceedings (Indian Academy of Sciences) - Mathematical Sciences, 127(3):411-421, 2017.

[10] M. Ddamulira, C. A. Gómez-Ruiz and F. Luca. On a problem of Pillai with $k$-generalized Fibonacci numbers and powers of 2. Monatshefte für Mathematik, 187(4):635-664, 2018.

[11] M. O. Hernane, F. Luca, S. E. Rihane and A. Togbé. On Pillai's problem with Pell numbers and powers of 2. Hardy-Ramanujan Journal, 41 :22-31, 2018.

[12] S. H. Hernndez, F. Luca and L. M. Rivera. On Pillais problem with the Fibonacci and Pell sequences Boletín de la Sociedad Matemática Mexicana, To appear.

[13] A. Dujella and A. Pethő. A generalization of a theorem of Baker and Davenport. The Quarterly Journal of Mathematics, 49(195):291-306, 1998.

[14] S. S. Gúzman and F. Luca. Linear combinations of factorials and $s$-units in a binary recurrence sequence. Annales Mathemántiques du Québec, 38:169-188, 2014.

[15] A. Herschfeld. The equation $2^{x}-3^{y}=d$. Bull. Amer. Math. Soc. , 41, pp. 631 (1935).

[16] A. Herschfeld. The equation $2^{x}-3^{y}=d$. Bull. Amer. Math. Soc. , 42, pp. 231-234 (1936).

[17] E. M. Matveev, An explicit lower bound for a homogeneous rational linear form in the logarithms of algebraic numbers, II, Izv. Ross. Akad. Nauk Ser. Mat. 64, No.6 , pp. 125-180 (2000); translation in Izv. Math. 64 , No. 6, pp. 1217-1269 (2000).

[18] P. Mihăilescu Primary cyclotomic units and a proof of Catalan's conjecture Journal für die reine und angewandte Mathematik (Crelles Journal), 2004.572 (2006): 167-195.

[19] S. S. Pillai. On $a^{x}-b^{y}=c$. J. Indian Math. Soc. (N.S.), 2: pp. 119-122 (1936).

[20] S. S. Pillai. A correction to the paper On $a^{x}-b^{y}=c$. J. Indian Math. Soc. (N.S.), 2, pp. 215, (1937).

MAHADi DDAMULiRa

Institute of Analysis and Number Theory, Graz University of Technology

KOPERNIKUSGASSE 24/II

A-8010 Graz, Austria

E-mail address: mddamulira@tugraz.at; mahadi@aims.edu.gh 